Global Journal of Mathematical Analysis, $7(1)(2019) 4-14$
Global Journal of Mathematical Analysis
WPC
Website: www.sciencepubco.com/index.php/GJMA
Research paper

\title{
Mathematical study of the small oscillations of a finite cylindrical column liquid-gas under zero gravity
}

\author{
Hilal Essaouini $^{1 *}$, Pierre Capodanno \\ ${ }^{1}$ UniversityAbdelmalekEssaâdi, Faculty ofSciences, M2SMER28/FS/05, 93030Mhannech II, BP : 2121, Tétouan, Morocco \\ ${ }^{2}$ UniversitédeFranche-Comté, $2 B$, Rue des jardins, F-25000 Besançon, France \\ *Corresponding authorE-mail:hilal_essaouini@yahoo.fr
}

\begin{abstract}
This paper deals with the mathematical study of the small motions of a system formed by a cylindrical liquid column bounded by two parallel circular rings and an internal cylindrical column constituted by a barotropic gas under zero gravity. From the equations of motion, the authors deduce a variational equation. Then, the study of the small oscillations depends on the coerciveness of a hermitian form that appears in this equation. It is proved that this last problem is reduced to an auxiliary eigenvalues problem. The discussion shows that, under a simple geometric condition, the problem is a classical vibration problem.
\end{abstract}

Keywords: Gas Dynamics; Free Boundaries; Capillarity (Surface Tension); Small Oscillations; Variational Method.

\section{Introduction}

The problem of the small oscillations of an incompressible inviscid liquid under zero gravity, in which the surface tensions determine the character of the motion, is very important in the experiments in space laboratories. It is well-known that, in fabrication process under microgravity conditions, such as crystal growth, the oscillations of the free liquid surface are often detrimental effect on the product.

This problem has been widely studied since many years by numerous researchers (Moiseyev 1968; Bauer 1982-1989-1993; Morand 1992; Kopachevskii 2001; Langbein 2002; Capodanno 2001-2003).

In this work, the authors study the small oscillations of a system formed by a cylindrical liquid column bounded by two parallel circular rings, the liquid being anchored at the external rim of the rings, and an internal cylindrical column constituted by a barotropic gas, under zero gravity, schematizing an air bubble inside the liquid. From the equations of motion in linear theory, they deduce a variational formulation of the problem. The study of the spectrum of the problem depends on a hermitian sesquilinear form that appears in the variational equation and that represents the virtual work of the surface tension forces. The authors prove that the study of the coerciveness of this form in a suitable Hilbert space is reduced to an auxiliary eigenvalues problem. After a careful discussion, they show that, if we discard translations of the gas column orthogonal to its axis, and under a very simple geometric condition, the problem is classical vibration problem.

\section{Position of the problem}

In the absence of gravity, in the equilibrium position, with respect to orthogonal axes $O x y z$, the system is formed by

i) An incompressible inviscid liquid occupying the domain $\Omega_{0}$ defined by $b \leq r=\sqrt{x^{2}+y^{2}} \leq a,-h \leq z \leq h$, whose the bases are bounded by the circles $c_{0}, c_{0}^{\prime}, c, c^{\prime}$. We denote by $S$ (resp. $S_{0}$ ) the surfaces $r=b,-h \leq z \leq h$ (resp. $r=a,-h \leq z \leq h$ ). The disks $C_{0}$ (resp. $\left.C_{0}^{\prime}\right)$ defined by $(z=h ; r \leq a),(z=-h r \leq a)$ are rigid.

ii) A barotropic gas, that occupies the domain $\Omega(r \leq b,-h \leq z \leq h)$.

We denote by $\vec{n}$ the unit vector normal to $S$ and $S_{0}$, directed to the exterior of the domains $\Omega$ and $\Omega_{0}$. We introduce the cylindrical coordinates $r, \theta, z$ and also $s=b \theta, s_{0}=a \theta$.

At the instant $t, S$ (resp. $S_{0}$ ) occupies the position $S_{t}$ (resp. $\left.S_{0 t}\right)$ defined by the equation $r=b+\zeta(s, z, t)\left(\operatorname{resp} . r=a+\zeta_{0}\left(s_{0}, z, t\right)\right)$. $\zeta, \zeta_{0}$ and their derivatives will be considered as quantities of the first order with respect to the amplitude of oscillations.

We denote by $\alpha$ (resp. $\beta$ ) the surface tension of $S_{0}$ (resp. $S$ ).

We suppose that: 


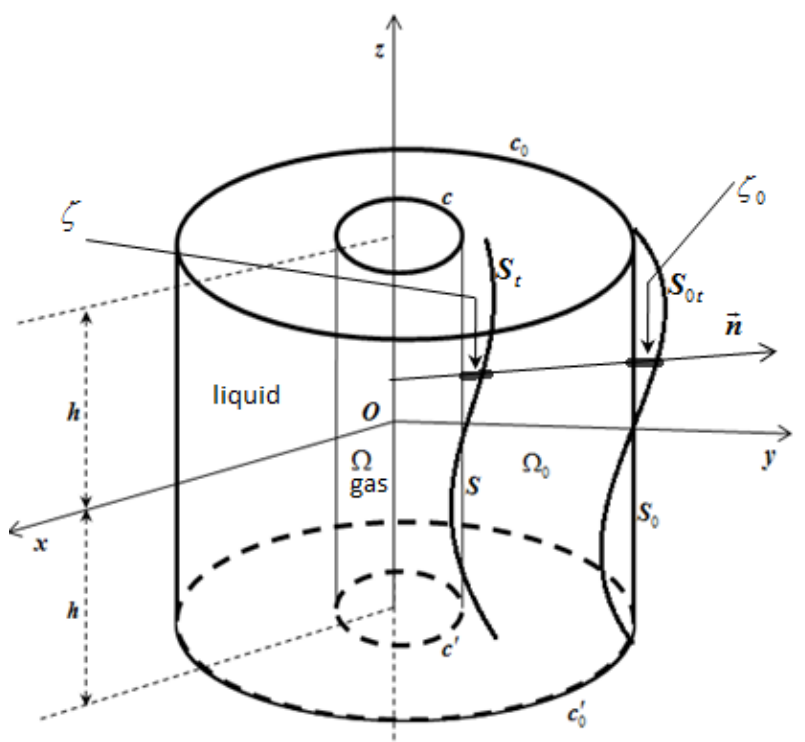

a) The liquid is anchored at the rims $c_{0}$ and $c_{0}^{\prime}$ of the disks $C_{0}$ and $C_{0}^{\prime}$, so that we have

$\zeta_{0}=0$ for $z= \pm h$

b) On the boundaries $c$ and $c^{\prime}$ takes place the condition: the contact angle is constant.

The angle of the normal to $S$ and the planes $z= \pm h$ along $c$ and $c^{\prime}$ is a right angle.

If $M$ is a point of $S_{t}$, its coordinates are

$$
x=[b+\zeta(s, z, t)] \cos \frac{s}{b} ; \quad y=[b+\zeta(s, z, t)] \sin \frac{s}{b} ; z=z
$$

The component on $O z$ of the normal vector $\frac{\partial \vec{M}}{\partial s} \times \frac{\partial \vec{M}}{\partial z}$ is equal to $-\frac{b+\zeta}{b} \zeta_{z}$, so that the condition is

$\zeta_{z}=0$ for $z= \pm h\left(\zeta_{z}=\frac{\partial \zeta}{\partial z}\right)$

We are going to study, in the framework of the linear theory, the small oscillations of the system about its equilibrium position.

\section{Equations of motion}

\subsection{Equations of the liquid}

If $\rho_{0}$ is its constant density, $P_{0}$ the pressure, $\vec{u}_{0}$ the small displacement of a particle from its equilibrium position, we have

$\rho_{0} \ddot{\vec{u}}=-\overrightarrow{\operatorname{grad}} P_{0}$ in $\Omega_{0}$ (Euler's equation)

$\operatorname{div} \dot{\vec{u}}_{0}=0$ in $\Omega_{0}$ (incompressibility)

From the last equation, we deduce by integrating between the date of the equilibrium and $t$

$\operatorname{div} \vec{u}_{0}=0$ in $\Omega_{0}$

The kinematic conditions are, (1) and

$u_{0 n \mid S_{0}} \stackrel{\text { def }}{=} \vec{u}_{0} \cdot \vec{n}_{\mid s_{0}}=\zeta$

$u_{0 z}=0$ for $z= \pm h, b \leq r \leq a$

In the equilibrium position, $P_{0}$ is a constant denoted by $P_{0 s t}$.

If the constant external atmospheric pressure is $p_{a}$, the Laplace law gives

During the motion, its gives

$$
P_{0 s t}=p_{a}+\frac{\alpha}{a}
$$

$$
P_{0 \mid \varsigma_{0}}-p_{a}=-\alpha\left(\frac{1}{R_{1}^{0}}+\frac{1}{R_{2}^{0}}\right),
$$


Where $R_{1}^{0}$ and $R_{2}^{0}$ are the principal radii of curvature of $S_{0 t}$, regarded as negative when the centre of curvature lies on the same side of the surface as the liquid.

The calculation of a circular cylinder is well-known (Lamb 1932) and we have

$$
P_{0 \mid S_{0 t}}-p_{a}=-\alpha\left[\frac{1}{a}+\left(\zeta_{0 s_{s_{0}}}+\zeta_{0 z z}+\frac{1}{a^{2}} \zeta_{0}\right)\right]
$$

Let us introduce the dynamic pressure $p_{0}$ by

$$
P_{0}=P_{0 s t}+p_{0}
$$

We have

$$
p_{0 \mid S_{0}}=-\alpha\left(\zeta_{0 s_{0} s_{0}}+\zeta_{0 z z}+\frac{1}{a^{2}} \zeta_{0}\right)
$$

\subsection{Equations of the gas}

Denoting by $\rho^{*}$ the density, $P^{*}$ the pressure, $\vec{u}$ the small displacement of a particle from its equilibrium position, we have in $\Omega$

$P^{*}=Q\left(\rho^{*}\right)$ (equation of state)

Where $Q$ is an increasing function of $\rho^{*}$,

$\rho^{*} \ddot{\vec{u}}=-\overrightarrow{\operatorname{grad}} P^{*}$ (Euler's equation)

$\frac{\partial \rho^{*}}{\partial t}+\operatorname{div}\left(\rho^{*} \dot{\vec{u}}\right)=0$ (continuity equation)

In the equilibrium position, $P^{*}$ is a constant denoted by $P$; then, $\rho^{*}$ is a constant denoted by $\rho$ and we have $P=Q(\rho)$. On the other hand, the Laplace law gives

$$
P_{\mid s}-P_{0 \mid s}=\frac{\beta}{b}
$$

and there fore

$$
P=p_{0}+\frac{\alpha}{a}+\frac{\beta}{b}
$$

We are going to linearize the equations of motion.

We set

$$
P^{*}=P+p+\ldots \quad ; \rho^{*}=\rho+\rho^{1}+\ldots
$$

Where the dynamic pressure $p$ and $\rho^{1}$ are of the first order and the dots represent terms of larger order.

The linearized continuity equation can be written

$$
\frac{\partial \rho^{1}}{\partial t}+\operatorname{div}(\rho \dot{\vec{u}})=0
$$

then, by integrating

the equation of state gives

$$
\rho^{1}=-\rho \operatorname{div} \vec{u}
$$

$$
\begin{aligned}
P+p+\ldots & =Q\left(\rho+\rho^{1}+\ldots\right) \\
& =Q(\rho)+Q^{\prime}(\rho) \rho^{1}+\ldots
\end{aligned}
$$

Setting classically $Q^{\prime}(\rho)=c^{2}$, here positive constant, we have

and then

$$
p=c^{2} \rho^{1}
$$

$p=-\rho c^{2} \operatorname{div} \vec{u}$

Since $\overrightarrow{\operatorname{grad}} P^{*}=\overrightarrow{\operatorname{grad}} p$, the linearized Euler's equation takes the form

$\ddot{\vec{u}}=c^{2} \overrightarrow{\operatorname{grad}}(\operatorname{div} \vec{u})$

The kinematic conditions are (2) and

$u_{n \mid s} \stackrel{\text { def }}{=} \vec{u} \cdot \vec{n}_{\mid s}=\zeta$

$u_{n}=0$ for $z= \pm h, r \leq b$

$u_{n \mid s}=u_{0 n \mid s}$ 
The dynamic condition on $S_{t}$ is given, with obvious notations, by the Laplace law

From which we deduce easily

$$
P_{\mid S_{t}}^{*}-P_{0 \mid S_{t}}=-\beta\left[-\frac{1}{b}+\zeta_{s s}+\zeta_{z z}+\frac{1}{b^{2}} \zeta\right]
$$

$p_{\mid s}-p_{0 \mid S}=-\beta\left(\zeta_{s s}+\zeta_{z z}+\frac{1}{b^{2}} \zeta\right)$

Finally, the volume of the liquid being constant, we must have

$$
\int_{S_{0}} \zeta_{0} d S_{0}-\int_{S} \zeta d S=0
$$

We remark that, if the conditions of the Lagrange's theorem are satisfied, $\vec{u}_{0}$ and $\vec{u}$ are gradients.

\section{Variational formulation of the problem}

We define the space of the kinematically admissible displacements $\overrightarrow{\tilde{u}}_{0}$ and $\overrightarrow{\tilde{u}}$ by

$$
\begin{gathered}
\operatorname{div} \overrightarrow{\tilde{u}}_{0}=0 \text { in } \Omega_{0} ; \tilde{u}_{0 n}=0 \text { on } z= \pm h, b \leq r \leq a ; \tilde{u}_{0 n \mid S_{0}}\left(=\tilde{\zeta}_{0}\right)=0 \text { on } c_{0}, c_{0}^{\prime} ; \\
\tilde{u}_{n}=0 \text { on } z= \pm h, r \leq b ; \tilde{u}_{0 n \mid S}=\tilde{u}_{n \mid S}(=\tilde{\zeta}) ; \\
\int_{S_{0}} \tilde{u}_{0 n \mid S_{0}} d S_{0}-\int_{S} \tilde{u}_{n \mid S} d S=0 .
\end{gathered}
$$

This will stated more precisely in what follows.

We have

$$
\int_{\Omega_{0}} \rho_{0} \ddot{\vec{u}}_{0} \cdot \overline{\overrightarrow{\tilde{u}}}_{0} d \Omega_{0}=-\int_{\Omega_{0}} \overrightarrow{\operatorname{grad}} p_{0} \cdot \overline{\overline{\tilde{u}}} d \Omega_{0}=-\int_{S_{0}} p_{0 \mid S_{0}} \overline{\tilde{u}}_{0 n \mid S_{0}} d S_{0}+\int_{S} p_{0 \mid s} \overline{\tilde{u}}_{0 n \mid S} d S
$$

By using the Green formula,

$$
\int_{\Omega} \rho \ddot{\vec{u}} \cdot \overline{\overline{\tilde{u}}} d \Omega=\int_{\Omega} \rho c^{2} \overrightarrow{\operatorname{grad}}(\operatorname{div} \vec{u}) \cdot \overline{\overrightarrow{\tilde{u}}} d \Omega=-\int_{S} p_{\mid s} \overline{\tilde{\tilde{u}}}_{n \mid S} d S-\rho c^{2} \int_{\Omega} \operatorname{div} \vec{u} \cdot \operatorname{div} \overline{\overline{\tilde{u}}} d \Omega,
$$

where we have used the Green formula and (11).

Adding and using the condition (7) and (16), we obtain

$$
\int_{\Omega_{0}} \rho_{0} \ddot{\vec{u}}_{0} \cdot \overline{\tilde{\tilde{u}}}_{0} d \Omega_{0}+\int_{\Omega} \rho \ddot{\vec{u}} \cdot \overline{\bar{u}} d \Omega+\rho c^{2} \int_{\Omega} \operatorname{div} \vec{u} \cdot \operatorname{div} \overline{\overline{\tilde{u}}} d \Omega-\beta \int_{S}\left(\zeta_{s s}+\zeta_{z z}+\frac{1}{b^{2}} \zeta\right) \cdot \overline{\tilde{\zeta}} d S-\alpha \int_{S_{0}}\left(\zeta_{o_{s o s} s_{0}}+\zeta_{0 z z}+\frac{1}{a^{2}} \zeta_{0}\right) \cdot \overline{\tilde{\zeta}} d S_{0}=0
$$

We are going to integrate by parts the last two integrals.

For instance, we have

$$
\int_{s_{0}} \zeta_{0 s_{0} s_{0}} \cdot \overline{\tilde{\zeta}} d S_{0}=\int_{-h}^{h}\left[\int_{0}^{2 \pi a} \zeta_{0 s_{s} s_{0}} \cdot \overline{\tilde{\zeta}_{0}} d s_{0}\right] d z=\int_{-h}^{h}\left\{\left[\zeta_{0 s_{0}} \cdot \overline{\tilde{\zeta}}_{0}\right]_{0}^{2 \pi a}-\int_{0}^{2 \pi a} \zeta_{0 s_{0}} \cdot \overline{\tilde{\zeta}}_{0 s_{0}} d s_{0}\right\} d z
$$

i.e., in accordance with the periodicity of $\zeta_{0}$ and $\tilde{\zeta}_{0}$ :

$$
\int_{S_{0}} \zeta_{0 s_{s_{0}} s_{0}} \cdot \overline{\tilde{\zeta}}_{0} d S_{0}=-\int_{S_{0}} \zeta_{0 s_{0}} \cdot \overline{\tilde{\zeta}}_{0 s_{0}} d S_{0}
$$

Finally, after analogous calculations, we obtain the variational equation of the problem

$\int_{\Omega_{0}} \rho_{0} \ddot{\vec{u}}_{0} \cdot \overline{\overrightarrow{\tilde{u}}}_{0} d \Omega_{0}+\int_{\Omega} \rho \ddot{\vec{u}} \cdot \overline{\overline{\tilde{u}}} d \Omega+\rho c^{2} \int_{\Omega} \operatorname{div} \vec{u} \cdot \operatorname{div} \overline{\overline{\tilde{u}}} d \Omega+\alpha \int_{S_{0}}\left(\zeta_{0 s_{0}} \overline{\tilde{\zeta}}_{s_{0}}+\zeta_{0 z} \overline{\tilde{\zeta}}_{0 z}-\frac{1}{a^{2}} \zeta_{0} \overline{\tilde{\zeta}}_{0}\right) d S_{0}+\beta \int_{s}\left(\zeta_{s} \overline{\tilde{\zeta}}_{s}+\zeta_{z} \overline{\tilde{\zeta}}-\frac{1}{b^{2}} \zeta \overline{\tilde{\zeta}}\right) d S=0$,

for all admissible $\overrightarrow{\tilde{u}}_{0}, \overrightarrow{\tilde{u}}$.

\section{Remark:}

It is easy to give a mechanical interpretation of the last two terms of the equation (18).

Indeed, it is well-known (Moiseyev 1968) that the virtual work of the surface tension forces is given by the formula

$$
\delta \Pi=\alpha \int_{S_{0}}\left\{\left(\frac{1}{R_{1}^{0}}+\frac{1}{R_{2}^{0}}\right) \tilde{u}_{0 n \mid S_{0}}\right\} d S_{0}+\beta \int_{S}\left\{\left(\frac{1}{R_{1}}+\frac{1}{R_{2}}\right) \tilde{u}_{n \mid S}\right\} d S
$$

Using the expressions of the mean curvatures and integrating by parts, we obtain the last two terms of (18). 


\section{Study of the bilinear form associated to surface-integrals}

5.1. We suppose that $\zeta_{0} \in H_{0}^{1}\left(S_{0}\right), U(.) \in V$ and setting

$$
Z=\left(\begin{array}{l}
\zeta_{0}=u_{0 n \mid s_{0}} \\
\zeta=u_{n \mid S}
\end{array}\right) \text {, that } Z \in \mathbb{N}^{1}=\left\{Z \in H_{0}^{1}\left(S_{0}\right) \oplus H^{1}(S) ; \int_{S_{0}} \zeta_{0} d S_{0}-\int_{S} \zeta d S=0\right\} \text {, }
$$

that is a Hilbert space for the norm defined by

We introduce the space

$$
\|Z\|_{\mathbb{s}^{\prime}}^{2}=\int_{S_{0}}\left(\zeta_{0 s_{0}}^{2}+\zeta_{0 z}^{2}+\zeta_{0}^{2}\right) d S_{0}+\int_{S}\left(\zeta_{s}^{2}+\zeta_{z}^{2}+\zeta^{2}\right) d S
$$

$$
\mathscr{S}^{2}=\left\{Z \in L^{2}\left(S_{0}\right) \oplus L^{2}(S) ; \int_{S_{0}} \zeta_{0} d S_{0}-\int_{S} \zeta d S=0\right\},
$$

equipped with the norm defined by

$$
\|Z\|_{2^{2}}^{2}=\frac{\alpha}{a^{2}} \int_{S_{0}} \zeta_{0}^{2} d S_{0}+\frac{\beta}{b^{2}} \int_{s} \zeta^{2} d S .
$$

The embedding from $\aleph^{1}$ into $\mathscr{L}^{2}$ is classically continuous, dense and compact.

We are going to study the bilinear form

$$
M(Z, \tilde{Z})=\alpha \int_{s_{0}}\left(\zeta_{0 s_{0}} \overline{\tilde{\zeta}}_{0 s_{0}}+\zeta_{0 z} \overline{\tilde{\zeta}}_{0 z}-\frac{1}{a^{2}} \zeta_{0} \overline{\tilde{\zeta}}_{0}\right) d S_{0}+\beta \int_{s}\left(\zeta_{s} \overline{\tilde{\zeta}}_{s}+\zeta_{z} \overline{\tilde{\zeta}}_{z}-\frac{1}{b^{2}} \zeta \bar{\zeta}\right) d S,
$$

on the space $\aleph^{1} \times \aleph^{1}$.

\subsection{This form is obviously symmetrical and continuous}

In order to study its coerciveness, we use a method that we can find in the book (Roseau 1984), so that we will sketch the proof. We set

$$
\lambda=\inf _{Z \in \mathrm{N}^{\prime}} \frac{\alpha \int_{S_{0}}\left(\zeta_{0 s_{0}}^{2}+\zeta_{0_{z}}^{2}\right) d S_{0}+\beta \int_{S}\left(\zeta_{s}^{2}+\zeta_{z}^{2}\right) d S}{\frac{\alpha}{a^{2}} \int_{S_{0}} \zeta_{0}^{2} d S_{0}+\frac{\beta}{b^{2}} \int_{S} \zeta^{2} d S}
$$

We can prove that there exist $\hat{Z} \in \aleph^{1}$ such that $\lambda$ is the value of the ratio for $Z=\hat{Z}$ and that $\lambda>0$.

By the definition of $\lambda$, we have

$$
\alpha \int_{S_{0}}\left(\zeta_{0 s_{0}}^{2}+\zeta_{0 z}^{2}\right) d S_{0}+\beta \int_{s}\left(\zeta_{s}^{2}+\zeta_{z}^{2}\right) d S-\lambda\left[\frac{\alpha}{a^{2}} \int_{s_{0}} \zeta_{0}^{2} d S_{0}+\frac{\beta}{b^{2}} \int_{S} \zeta^{2} d S\right] \geq 0 \quad \forall Z \in \aleph^{1}(19)
$$

Setting

$$
Z=\hat{Z}+\varepsilon \delta Z, \varepsilon \in I R, \delta Z=\left(\begin{array}{l}
\delta \zeta_{0} \\
\delta \zeta
\end{array}\right) \in \aleph^{\prime \prime}
$$

we see that this inequality is possible for each $\varepsilon \in \mathbb{R}$ only if the coefficient of $2 \varepsilon$ is equal to zero

$$
\forall \delta \zeta_{0} \in H_{0}^{1}\left(S_{0}\right), \forall \delta \zeta \in H^{1}(S), \int_{S_{0}} \delta \zeta_{0} d S_{0}-\int_{S} \delta \zeta d S=0 .
$$

Introducing a multiplier $\mu$ associated to the last condition, we obtain

$$
\int_{S_{0}}\left\{\alpha\left(\hat{\zeta}_{0 s_{0}} \delta \zeta_{0 s_{0}}+\hat{\zeta}_{0 z} \delta \zeta_{0 z}-\frac{\lambda}{a^{2}} \hat{\zeta}_{0} \delta \zeta_{0}\right)-\mu \delta \zeta_{0}\right\} d S_{0}+\int_{s}\left\{\beta\left(\hat{\zeta}_{s} \delta \zeta_{s}+\hat{\zeta}_{z} \delta \zeta_{z}-\frac{\lambda}{b^{2}} \hat{\zeta} \delta \zeta\right)+\mu \delta \zeta\right\} d S=0
$$

$\forall \delta \zeta_{0} \in H_{0}^{1}\left(S_{0}\right), \forall \delta \zeta \in H^{1}(S)$

Since $\mathscr{D}\left(S_{0}\right) \subset H_{0}^{1}\left(S_{0}\right), \mathscr{D}(S) \subset H^{1}(S)$, the equation (20) is valid for each $\delta \zeta_{0} \in \mathscr{D}\left(S_{0}\right), \delta \zeta \in \mathscr{D}(S)$, so that we have

$\hat{\zeta}_{0 s_{s} s_{0}}+\hat{\zeta}_{0 z z}+\frac{\lambda}{a^{2}} \hat{\zeta}_{0}+\frac{\mu}{\alpha}=0$ in $\mathscr{D}^{\prime}\left(S_{0}\right)$

$\hat{\zeta}_{s s}+\hat{\zeta}_{z z}+\frac{\lambda}{b^{2}} \hat{\zeta}-\frac{\mu}{\beta}=0$ in $\mathscr{D}^{\prime}(S)$

And by virtue of a Schwartz theorem on the elliptic equation (Schwartz 1966), in the classical sense. Therefore, the solutions of (21), (22) are $C^{\infty}$.

Turning to the equation (20), we can integrate by parts; we find easily

$$
\int_{s_{0}}\left\{\alpha\left[-\hat{\zeta}_{0 s s_{0}}-\hat{\zeta}_{0 z z}-\frac{\lambda}{a^{2}} \hat{\zeta}_{0}\right]-\mu\right\} \delta \zeta_{0} d S_{0}+\int_{s}\left\{\beta\left[-\hat{\zeta}_{s s}-\hat{\zeta}_{z z}-\frac{\lambda}{b^{2}} \hat{\zeta}\right]+\mu\right\} \delta \zeta d S-\int_{0}^{2 \pi b}\left[\hat{\zeta}_{z}(s, h) \delta \zeta(s, h)-\hat{\zeta}_{z}(s,-h) \delta \zeta(s,-h)\right] d s=0
$$


$\forall \delta \zeta_{0} \in H_{0}^{1}\left(S_{0}\right), \forall \delta \zeta \in H^{1}(S)$

The first line is equal to zero by virtue of the equations (21) and (22).

$\delta \zeta(s, h)$ and $\delta \zeta(s,-h)$ being arbitrary in $L^{2}(0,2 \pi b)$, we obtain

$$
\hat{\zeta}_{z}=0 \text { for } z= \pm h
$$

Eliminating $\lambda$ by using the condition (17), we obtain

$$
\mu=-k_{0} a^{2} \int_{0}^{2 \pi a}\left[\hat{\zeta}_{0 z}\left(s_{0}, h\right)-\hat{\zeta}_{0 z}\left(s_{0},-h\right)\right] d s_{0}, \text { with } k_{0}=\frac{1}{4 \pi h\left(\frac{a^{3}}{\alpha}+\frac{b^{3}}{\beta}\right)} .
$$

Finally, we obtain $\inf _{N^{\prime}} \lambda$ by solving the eigenvalues problem $P_{\lambda}$ :

$\zeta_{0 s_{0} s_{0}}+\zeta_{0 z z}+\frac{\lambda}{a^{2}} \zeta_{0}-\frac{k_{0}}{\alpha} a^{2} \int_{0}^{2 \pi a}\left[\zeta_{0 z}\left(s_{0}, h\right)-\zeta_{0 z}\left(s_{0},-h\right)\right] d s_{0}=0$

$\zeta_{s s}+\zeta_{z z}+\frac{\lambda}{b^{2}} \zeta+\frac{k_{0}}{\beta} a^{2} \int_{0}^{2 \pi a}\left[\zeta_{0 z}\left(s_{0}, h\right)-\zeta_{0 z}\left(s_{0},-h\right)\right] d s_{0}=0$

$\zeta_{0}(\operatorname{resp} \zeta) 2 \pi a-$ periodic in $s_{0}(\operatorname{resp} 2 \pi b-$ periodic in $s)$

$\zeta_{0}\left(s_{0} ; \pm h\right)=0\left(0 \leq s_{0} \leq 2 \pi a\right)$

$\zeta_{z}(s ; \pm h)=0(0 \leq s \leq 2 \pi b)$

$\int_{S_{0}} \zeta_{0} d S_{0}-\int_{S} \zeta d S=0$

and $\lambda$ is the smallest eigenvalue of this problem.

\subsection{For solving the problem $P_{\lambda}$, we use the method of separation of variables}

We seek the solutions in the form

$$
\zeta_{0}=S_{0}\left(s_{0}\right) Z_{0}(z) ; \zeta=S(s) Z(z) .
$$

Carrying out in the equations (23), (24), we obtain

$$
\begin{aligned}
& S_{0}^{\prime \prime} Z_{0}+S_{0} Z_{0}^{\prime \prime}+\frac{\lambda}{a^{2}} S_{0} Z_{0}-\frac{k_{0}}{\alpha} a^{2} \int_{0}^{2 \pi a} S_{0}\left(s_{0}\right) d s_{0}\left[Z_{0}^{\prime}(h)-Z_{0}^{\prime}(-h)\right]=0 \\
& S^{\prime \prime} Z+S Z^{\prime \prime}+\frac{\lambda}{b^{2}} S Z+\frac{k_{0}}{\beta} a^{2} \int_{0}^{2 \pi a} S_{0}\left(s_{0}\right) d s_{0}\left[Z_{0}^{\prime}(h)-Z_{0}^{\prime}(-h)\right]=0
\end{aligned}
$$

and the conditions (1), (2), (17) give

$$
Z_{0}( \pm h)=0
$$

$Z_{z}( \pm h)=0$

$\int_{0}^{2 \pi a} S_{0}\left(s_{0}\right) d s_{0} \cdot \int_{-h}^{h} Z_{0}(z) d z=\int_{0}^{2 \pi b} S(s) d s \cdot \int_{-h}^{h} Z(z) d z$

The discussion is long, but not difficult, so that we sketch it. We must distinguish four case.

- $\quad$ Case I: $\int_{0}^{2 \pi a} S_{0}\left(s_{0}\right) d s_{0}=0 ; \int_{0}^{2 \pi b} S(s) d s=0$

The condition (25) is satisfied and we deduce easily from the equation (23'), (24')

$$
\left\{\begin{array}{l}
S_{0}^{\prime \prime} Z_{0}+S_{0}\left(Z_{0}^{\prime \prime}+\frac{\lambda}{a^{2}} Z_{0}\right)=0 ; \quad Z_{0}( \pm h)=0 \\
S^{\prime \prime} Z+S\left(Z^{\prime \prime}+\frac{\lambda}{b^{2}} Z\right)=0 ; Z_{z}( \pm h)=0
\end{array}\right.
$$

a) At first, we study the problem for the pair $\left(S_{0}, Z_{0}\right)$.

We have

$$
-\frac{S_{0}^{\prime \prime}}{S_{0}}=\frac{Z_{0}^{\prime \prime}+\frac{\lambda}{a^{2}} Z_{0}}{Z_{0}}=\text { constant }=\frac{n^{2}}{a^{2}}, n \text { integer } \geq 1, \text { by virtue of the periodicity of } S_{0}\left(s_{0}\right) .
$$

At first, we obtain

$$
S_{0}\left(s_{0}\right)=A_{0 n} \cos \frac{n}{a} s_{0}+B_{0 n} \sin \frac{n}{a} s_{0}\left(A_{0 n}, B_{0 n} \text { constants }\right)
$$

The problem for $Z_{0}$ is possible only if $\lambda-n^{2}>0$. Setting $\lambda=n^{2}+m^{2} a^{2}$, we have the well-known eigenvalues problem 
The boundaryconditions give

$$
Z_{0}^{\prime \prime}+m^{2} Z_{0}=0 ; \quad Z_{0}( \pm h)=0
$$

$$
m=\frac{k \pi}{h} ; m=\frac{(2 k-1) \pi}{2 h}(k=1,2, \ldots)
$$

so, we have obtained a set of eigenvalues of the problem $P_{\lambda}$ :

$$
\lambda=n^{2}+\frac{k^{2} \pi^{2} a^{2}}{h^{2}} ; \lambda=n^{2}+\frac{(2 k-1)^{2} \pi^{2} a^{2}}{4 h^{2}}(n=1,2, \ldots ; k=1,2, \ldots)
$$

These eigenvalues are double and strictly greater than 1 .

The corresponding eigenfunctions of $P_{\lambda}$ are

$$
\begin{gathered}
\cos \frac{n s_{0}}{a} \sin \frac{k \pi}{h} z ; \sin \frac{n s_{0}}{a} \sin \frac{k \pi}{h} z, \\
\cos \frac{n s_{0}}{a} \cos \frac{(2 k-1) \pi}{2 h} z ; \sin \frac{n s_{0}}{a} \cos \frac{(2 k-1) \pi}{2 h} z .
\end{gathered}
$$

i) Now, we study the problem for the pair $(S, Z)$.

Like above, we obtain

$$
S(s)=A_{n} \cos \frac{n}{b} s_{0}+B_{n} \sin \frac{n}{b} s_{0}(n=1,2, \ldots)
$$

Setting $\lambda=n^{2}+m^{\prime 2} b$, we have for $Z(z)$ the eigenvalues problem

At first, we consider the case $m^{\prime}=0$.

$$
Z^{\prime \prime}+m^{\prime 2} Z=0 ; \quad Z^{\prime}( \pm h)=0
$$

For the problem $P_{\lambda}$, we have the double eigenvalues $\lambda=n^{2}$ and the eigenfunctions $\sin \frac{n}{b} s, \cos \frac{n}{b} s(n=1,2, \ldots)$.

But,here, we remark that $\zeta_{s s}+\zeta_{z z}+\frac{\lambda}{b^{2}} \zeta$ is equal to zero if we replace $\zeta$ by $\sin \frac{n}{b}, \cos \frac{n}{b}$. If these functions are solutions of the problem, we must discard them, because we are no longer in the framework of the linearization.

The case $m^{\prime} \neq 0$ can be treated like above and we obtain the set of eigenvalues of $P_{\lambda}$

$$
\lambda=n^{2}+\frac{k^{2} \pi^{2} b^{2}}{h^{2}} ; \lambda=n^{2}+\frac{(2 k-1)^{2} \pi^{2} b^{2}}{4 h^{2}},(n=1,2, \ldots ; k=1,2, \ldots)
$$

Which are double and strictly greater than 1 .

- $\quad$ Case II: $\int_{0}^{2 \pi a} S_{0}\left(s_{0}\right) d s_{0} \neq 0 ; \int_{0}^{2 \pi b} S(s) d s=0$

It is easy to see that $S_{0}\left(s_{0}\right)=$ constant and that, for $Z(z)$, we have the problem:

While the condition (25) gives

$$
Z_{0}^{\prime \prime}+\frac{\lambda}{a^{2}} Z_{0}=\frac{2 \pi a^{3} k_{0}}{\alpha}\left[Z_{0}^{\prime}(h)-Z_{0}^{\prime}(-h)\right] ; Z_{0}( \pm h)=0
$$

$$
\int_{-h}^{h} Z_{0}(z) d z=0
$$

Integrating the differential equation from $-h$ to $h$, we obtain

$$
\left(1-\frac{4 \pi a^{3} k_{0} h}{\alpha}\right)\left[Z_{0}^{\prime}(h)-Z_{0}^{\prime}(-h)\right]=0,
$$

from which we deduce, using the definition of $k_{0}$ :

$$
Z_{0}^{\prime}(h)-Z_{0}^{\prime}(-h)=0
$$

Finally, we have well-known eigenvalues problem

$$
Z_{0}^{\prime \prime}+\frac{\lambda}{a^{2}} Z_{0}=0 ; Z_{0}( \pm h)=0 ; \int_{-h}^{h} Z_{0}(z) d z=0
$$

The eigenvalues are $\lambda=\frac{k^{2} \pi^{2} a^{2}}{h^{2}}$, the eigenfunctions $\sin \frac{k \pi}{h} z(k=1,2, \ldots)$. 
Since $S_{0}=$ constant, they are the corresponding eigenvalues and eigenfunctions of $P_{\lambda}$. Obviously, the smallest eigenvaluest is $\frac{\pi^{2} a^{2}}{h^{2}}$.

It is easy to see that the problem for the pair $(S, Z)$ is the problem treated in the case I and, consequently doesnt introduce new eigenvalues.

- $\quad$ Case III: $\int_{0}^{2 \pi a} S_{0}\left(s_{0}\right) d s_{0}=0 ; \int_{0}^{2 \pi b} S(s) d s \neq 0$

At first, we consider the problem for the pair $(S, Z)$.

We find easily

$$
Z^{\prime \prime}+\frac{\lambda}{b^{2}} Z=0 ; Z^{\prime}( \pm h)=0
$$

and $S(s)=$ constant .

Finally, for the problem $P_{\lambda}$, the eigenvalues are

The associated eigenfunctions being

$$
\lambda=\frac{k^{2} \pi^{2} b^{2}}{h^{2}} ; \lambda=\frac{(2 k-1)^{2} \pi^{2} b^{2}}{4 h^{2}}(k=1,2, \ldots),
$$

$$
\cos \frac{k \pi}{h} z ; \sin \frac{(2 k-1) \pi}{2 h} z
$$

The smallest eigenvalue is obviously $\frac{\pi^{2} b^{2}}{4 h^{2}}$.

Now, we consider the problem for the pair $\left(S_{0}, Z_{0}\right)$.

We obtain

$$
-\frac{S_{0}^{\prime \prime}}{S_{0}}=\frac{Z_{0}^{\prime \prime}+\frac{\lambda}{a^{2}} Z_{0}}{Z_{0}}=\text { constant }=\frac{n^{2}}{a^{2}}(n=1,2, \ldots)
$$

This problem was solved in the case I; therefore, it dosent introduce new eigenvalues.

- Case IV: $\int_{0}^{2 \pi a} S_{0}\left(s_{0}\right) d s_{0} \neq 0 ; \int_{0}^{2 \pi b} S(s) d s \neq 0$

Let us consider the problem for the pair $(S, Z)$.

The equation (24') gives

$$
Z^{\prime \prime}+\frac{\lambda}{b^{2}} Z=\text { constant }, \text { denoted by } C,
$$

and if $S(s) \neq$ constant .

$$
\frac{S^{\prime \prime}}{S-\frac{1}{2 \pi b} \int_{0}^{2 \pi b} S(s) d s}=-\frac{C}{Z}=\text { constant } .
$$

The case $Z=$ Cte being possible since $Z^{\prime}( \pm h)=0$, we must distinguish two cases.

a) $\quad S(s) \neq$ constant,$Z=$ constant

Then we have

$$
S^{\prime \prime}+\frac{\lambda}{b^{2}}\left(S-\frac{1}{2 \pi b} \int_{0}^{2 \pi b} S(s) d s\right)=0 .
$$

Because the periodicity, we must have $\lambda=n^{2}(n=1,2, \ldots)$ and we obtain

$$
S(s)=A_{n} \cos \frac{n}{b} s+B_{n} \sin \frac{n}{b} s+D ; D=\frac{1}{2 \pi b} \int_{0}^{2 \pi b} S(s) d s
$$

Consequently, for the problem $P_{\lambda}$, we have the eigenfunctions

$$
\zeta=\cos \frac{n}{b} s \quad ; \zeta=\sin \frac{n}{b} s \quad(n=1,2, \ldots)
$$

The eigenvalues being $\lambda=n^{2}$.

These results were found in like case I ; therefore, we don't obtain new eigenvalues.

Obviously, in accordance with a previous remark, if $\cos \frac{s}{b}, \sin \frac{s}{b}$ are solutions of the problem, we must discard them.

b) $\quad S(s)=$ constant . It is easy to prove that $S_{0}$ is constant and the condition (25) gives

$$
2 \pi a S_{0} \int_{0}^{2 \pi a} Z_{0} d z=2 \pi b S \int_{0}^{2 \pi b} Z d z
$$


$S_{0}$ and $S$ being arbitrary, we have

$$
\int_{0}^{2 \pi a} Z_{0} d z=0 ; \int_{0}^{2 \pi b} Z d z=0,
$$

and we obtain the following problems

$$
\begin{gathered}
Z_{0}^{\prime \prime}+\frac{\lambda}{a^{2}} Z_{0}=0 ; Z_{0}( \pm h)=0 ; \int_{0}^{2 \pi a} Z_{0} d z=0 ; \\
Z^{\prime \prime}+\frac{\lambda}{b^{2}} Z=0 ; Z^{\prime}( \pm h)=0 ; \int_{0}^{2 \pi a} Z d z=0
\end{gathered}
$$

The first was solved in the case II, the second in the case III.

They don't introduce new eigenvalues.

5.4. By virtue of the results that we have obtained for the eigenfunctions of the problems concerningthe pairs $(S, Z),\left(S_{0}, Z_{0}\right)$, we found,by the method of separation of variables, all the eigenfunctions and all the eigenvalues of the problem $P_{\lambda}$.

5.5. Since $a>b$ the smallest eigenvalues $\frac{\pi^{2} a^{2}}{h^{2}}, \frac{\pi^{2} b^{2}}{4 h^{2}}$ are strictly greater than 1 if $h<\frac{\pi b}{2}$

5.6. On the other hand, we must discard the solutions $\zeta=\cos \frac{s}{b}, \zeta=\sin \frac{s}{b}$ corresponding to the eigenvalue $\lambda=1$.

But we can give a mechanical interpretation of these solutions: they correspond to small translations without deformation of the internal cylinder along the axes $O x, O y$.

For instance, $\zeta=\xi \cos \frac{s}{b}(|\xi|$ small $)$ corresponds to a displacement $\xi \vec{x}$ along $O x$.

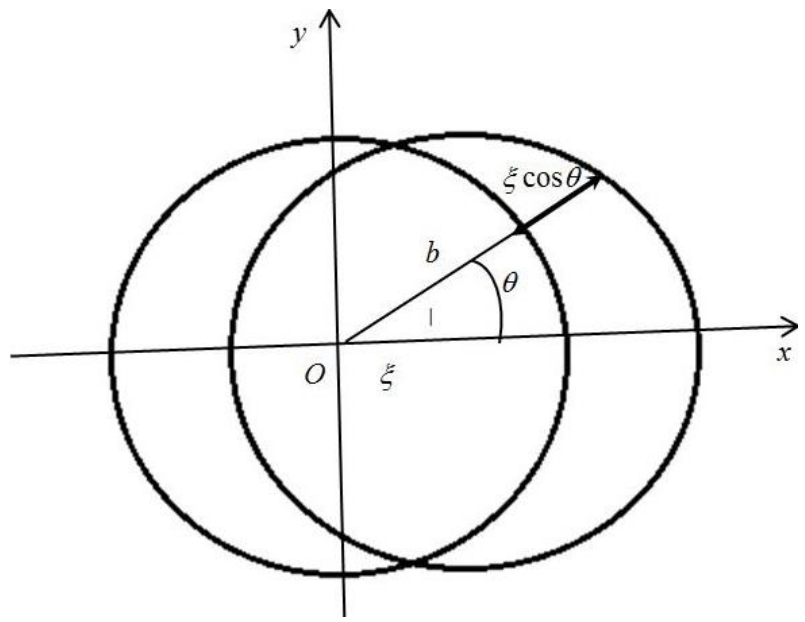

Indeed, the equation of the new cylinder is

or, neglecting the terms of the second order

$$
(x-\xi)^{2}+y^{2}=b^{2},
$$

and finally

$$
r^{2}-2 \xi r \cos \theta-b^{2}=0
$$

$$
r=b+\xi \cos \theta
$$

\subsection{Therefore, we introduce the space}

$$
\aleph^{0}=\left\{\aleph \in \aleph^{11} ; \int_{s} \zeta \cos \frac{s}{b} d S=0 ; \int_{s} \zeta \sin \frac{s}{b} d S=0\right\}
$$

Then, if $h<\frac{\pi b}{2}$, we have

$$
\lambda^{\prime}=\inf _{Z \in \mathbb{N}^{\circ}} \frac{\alpha \int_{S_{0}}\left(\zeta_{0 s_{0}}^{2}+\zeta_{0_{z}}^{2}\right) d S_{0}+\beta \int_{s}\left(\zeta_{s}^{2}+\zeta_{z}^{2}\right) d S}{\frac{\alpha}{a^{2}} \int_{S_{0}} \zeta_{0}^{2} d S_{0}+\frac{\beta}{b^{2}} \int_{S} \zeta^{2} d S}>1
$$

We are going to prove that the bilinear form $M(Z, \tilde{Z})$, obviously symmetrical and continuous on $\aleph^{0} \times \aleph^{0}$, is $\aleph^{0}-$ coercive. Indeed, with $0<\varepsilon<1$, we have by using the precedent inequality: 


$$
M(Z, Z) \geq \varepsilon\left[\alpha \int_{S_{0}}\left(\zeta_{0 s_{0}}^{2}+\zeta_{0 z}^{2}\right) d S_{0}+\beta \int_{S}\left(\zeta_{s}^{2}+\zeta_{z}^{2}\right) d S\right]+\left[(1-\varepsilon) \lambda^{\prime}-1\right]\left[\frac{\alpha}{a^{2}} \int_{S_{0}} \zeta_{0}^{2} d S_{0}+\frac{\beta}{b^{2}} \int_{S} \zeta^{2} d S\right]
$$

Choosing $0<\varepsilon<1-\lambda^{\prime-1}$, we see that $M(.,$.$) is \aleph^{0}-$ coercive. Consequently, $[M(Z, \tilde{Z})]^{1 / 2}$ define on $\aleph^{0}$ a norm that is equivalent to the norm of $\aleph^{0}$ or $\aleph^{1}$.

\section{The problem is a classical vibration problem}

i) Let us introduce the space

$$
V=\left\{\begin{array}{l}
U=\left(\begin{array}{l}
\vec{u}_{0} \\
\vec{u}
\end{array}\right) ; \\
\vec{u}_{0}=\overrightarrow{\operatorname{grad}} \varphi_{0} ; \varphi_{0} \in \tilde{H}^{1}\left(\Omega_{0}\right) \stackrel{\text { def }}{=}\left\{\varphi_{0} \in H^{1}\left(\Omega_{0}\right) ; \int_{\Omega_{0}} \varphi_{0} d \Omega_{0}=0\right\} ; \operatorname{div} \overrightarrow{\tilde{u}}_{0}=0 ; u_{0 n}=0 \text { for } z= \pm h ; b \leq r \leq a ; \\
\vec{u}=\overrightarrow{\operatorname{grad}} \varphi ; \varphi \in \tilde{H}^{1}(\Omega) \stackrel{\text { def }}{=}\left\{\varphi \in H^{1}(\Omega) ; \int_{\Omega} \varphi d \Omega=0\right\} ; u_{n}=0 \text { for } z= \pm h ; r \leq b ; u_{0 n \mid s}=u_{n \mid s} ; \quad Z=\left(\begin{array}{c}
u_{0 n \mid S_{0}}=\left.\frac{\partial \varphi_{0}}{\partial n}\right|_{S_{0}} \\
u_{n \mid s}=\left.\frac{\partial \varphi}{\partial n}\right|_{s}
\end{array}\right\} \in \aleph^{\aleph 0}
\end{array}\right\}
$$

equipped with the hilbertian norm defined by

$$
\|U\|_{v}^{2}=\int_{\Omega_{0}} \rho_{0}\left|\vec{u}_{0}\right|^{2} d \Omega_{0}+\int_{\Omega} \rho|\vec{u}|^{2} d \Omega+M(Z, Z)+\int_{\Omega}|\operatorname{div} \vec{u}|^{2} d \Omega,
$$

And $H$ completion of $V$ for the norm associated with the scalar product

$$
(U, \tilde{U})_{H}=\int_{\Omega_{0}} \rho_{0} \vec{u}_{0} \cdot \overline{\overrightarrow{\tilde{u}}} d \Omega_{0}+\int_{\Omega} \rho \vec{u} \cdot \overline{\overrightarrow{\tilde{u}}} d \Omega
$$

Setting

$$
a(U, \tilde{U})=M(Z, \tilde{Z})+\rho c^{2} \int_{\Omega} \operatorname{div} \vec{u} \cdot \operatorname{div} \overline{\overline{\tilde{u}}} d \Omega,
$$

we obtain the variational equation of the problem:

To find $U(.) \in V$ such that

$(\ddot{U}, \tilde{U})_{H}+a(U, \tilde{U})=0 \quad \forall \tilde{U} \in V$

ii) For proving that it is a matter of a classical vibration problem, it is sufficient to use the method introduced by (Sanchez Hubert 1989 : pp 65-68),we must prove that:

a) $[a(U, U)]^{1 / 2}$ defines on $V$ a norm equivalent to $\|U\|_{V}$.

b) The embedding, $V \subset H$ obviously dense and continuous, is compact.

We omit the proof that is analogous to the proof in (Sanchez Hubert 1989), distinguishing here obviously the problem for $\varphi_{0}$ and the problem for $\varphi$.

c) Finally, if we discard both solutions that correspond to displacements without deformation of the internal cylinder along the axes $O x$ and $O y$, the problem (26) is a classical problem of vibration(Sanchez Hubert 1989).

There is a denumerable set of positive real eigenvalues $\omega_{n}^{2}$ such that

$0<\omega_{1}^{2} \leq \omega_{2}^{2} \leq \ldots \leq \omega_{n}^{2} \leq \ldots, \omega_{n}^{2} \rightarrow+\infty$ when $n \rightarrow+\infty$

The eigenelements $\left\{U_{n}\right\}$ form an orthogonal basis in $H$ and an orthogonal basis in $V$ equipped with the scalar product $a(U, U)$.

\section{Acknowledgement}

The authors are grateful to the referee and the editorial board for some useful comments that Improved the presentation of the paper.

\section{References}

[1] Bauer, H.F. Oscillations of immiscible liquids in free space or in spherical containers in zero gravity environnement. Ingenieur. Archiv. 363381(1982).https://doi.org/10.1007/BF00536679.

[2] Bauer, H.F. Natural frequencies and stability circular cylindrical immiscible systems, Appl. Micrograv. Technol. 27-44 (1989).

[3] Bauer, H.F. Natural frequencies and response of spinning liquid column with apparently sliding contact line, Acta. Mech. 115-122 (1993).https://doi.org/10.1007/BF01173146. 
[4] Capodanno, P. The stability of a configuration of a liquid layer in an axially symmetric container uniformly rotating under conditions of weightlesness, Prikladnaya Matematikai Mekhanika (П .M.M). 65(4). 619-630 (2001) (in Russian). English translation ; J. Appl. Math. Mech. 65(4). 605-616 (2001). https://doi.org/10.1016/S0021-8928(01)00065-X.

[5] Capodanno, P. Etude mathématique des petites oscillations d'un pont liquide sphérique entre deux membranes circulaires en apesanteur (in french). Rev. Roum. Math. Pure. Appl, 46. 207-224 (2001).

[6] Capodanno, P. The stability and the small oscillations of an onduloïdal fluid bridge between two coaxial disks under conditions of weightlessness. Prikladnaya Matematikai Mekhanika (П .M.M). 67(2). 283-299 (2003). (In Russian). English translation; J. Appl. Math. Mech. 67(2). 253-268 (2003).https://doi.org/10.1016/S0021-8928(03)90012-8.

[7] Kopachevskii, N.D., Krein, S.G. Operator approach to linear problems of hydrodynamics. Vol. 1, Birkhauser, Basel (2001).https://doi.org/10.1007/978-3-0348-8342-9 1.

[8] Lamb, H.: Hydrodynamics. Cambridge at the University Press, Cambridge (1932).

[9] Langbein, D. Capillary Surfaces-Shape- Stability- Dynamics, in particular under weightlessness. In: Springer Tracts in modern Physics. SpringerVerlag, Berlin (2002).https://doi.org/10.1007/3-540-45267-2.

[10] Moiseyev, N.N., Rumyantsev, V.V. Dynamic stability of bodies containing fluid. Springer- Verlag, Berlin (1968).https://doi.org/10.1007/978-3642-86452-0.

[11] Morand, H.J-P., Ohayon, R. Interactions fluides-structures. Masson, Paris (1992).

[12] Roseau, M. Vibrations des systèmes mécaniques. Masson, Paris (1984).

[13] Sanchez H.J., Sanchez P.E. Vibration and coupling of continuous systems. Asymptotic methods. Springer-Verlag, Berlin (1989).

[14] Schwartz, L. Théorie des distributions. Hermann, Paris (1966). 